Meta

Journal des traducteurs

Translators' Journal

\title{
La terminotique en interprétation de conférence : un potentiel à exploiter
}

\section{Daniel Gile}

Volume 32, numéro 2, juin 1987

Vers l'an 2000. La terminotique, bilan et prospectives

Objectives: Year 2000 Terminotics. State of the Art, Prospects for the

Future

URI : https://id.erudit.org/iderudit/002904ar

DOI : https://doi.org/10.7202/002904ar

Aller au sommaire du numéro

Éditeur(s)

Les Presses de l'Université de Montréal

ISSN

0026-0452 (imprimé)

1492-1421 (numérique)

Découvrir la revue

Citer cet article

Gile, D. (1987). La terminotique en interprétation de conférence : un potentiel à exploiter. Meta, 32(2), 164-169. https://doi.org/10.7202/002904ar d'utilisation que vous pouvez consulter en ligne.

https://apropos.erudit.org/fr/usagers/politique-dutilisation/ 


\title{
LA TERMINOTIQUE EN INTERPRÉTATION DE CONFÉRENCE : UN POTENTIEL À EXPLOITER
}

\author{
DANIEL GILE \\ INALCO, Paris, France
}

\section{LES TERMES TECHNIQUES EN INTERPRÉTATION DE CONFÊRENCE}

La traduction humaine commence par l'analyse de l'énoncé en langue de départ. Cette analyse permet au traducteur (ou à l'interprète) de se représenter le sens de l'énoncé sous forme de réseau sémantique, c'est-à-dire un réseau de relations logiques et fonctionnelles (causalité, égalité, appartenance, équivalence, etc.) qui relient entre eux des concepts (objets, idées, actions, noms de personnes, de lieux, chiffres, etc.) - en général, ces concepts sont désignés dans l'énoncé par des noms et groupes nominaux. Dans un deuxième temps, le traducteur reformule les idées en langue d'arrivée en fonction de ce réseau sémantique mais indépendamment de la structure linguistique de l'énoncé original, ce qui lui permet de s'exprimer avec une certaine liberté tout en restant fidèle à l'orateur (Gile 1985a).

Si la syntaxe du discours parlé est généralement simple et ne pose pas de problèmes de compréhension ou de réexpression des relations logiques et fonctionnelles du réseau sémantique, les concepts que relient celles-ci peuvent être difficiles à cerner à travers les termes en langue de départ ou à restituer en langue d'arrivée pour le profane.

Contrairement aux conférences internationales d'il y a vingt ans et plus, les réunions spécialisées avec interprétation des années 70 et 80 sont souvent d'une très grande technicité, et comportent des termes techniques à fortes doses. Les interprètes ne sont que rarement de véritables spécialistes du domaine qu'ils interprètent et se heurtent donc constamment à des difficultés terminologiques.

Sur le plan stratégique, les problèmes sont atténués par un travail de préparation consistant à apprendre ou à noter le plus grand nombre possible de termes et d'" équivalents " avant la conférence (Gile 1985c), étant entendu que dans une réunion technique où l'information est dense et quantitativement importante, la préparation ne peut être que partiellement efficace. Sur le plan tactique, divers expédients permettent de limiter les pertes d'information pour les auditeurs quand survient un terme que l'interprète ne comprend pas ou dont il ne connaît pas l'" équivalent » idoine (Gile 1985b). Il est toutefois clair que l'optimisation de la restitution des concepts véhiculés par les termes techniques passe par l'amélioration de la connaissance du lexique pertinent et par l'accès rapide aux termes inconnus en cabine.

\section{LE VOCABULAIRE SPÉCIALISÉ DES CONFÉRENCES}

Deux paramètres déterminants distinguent les besoins terminologiques des interprètes de ceux des traducteurs :

1. Le registre du discours oral, qui est généralement plus souple que celui du texte écrit, d'où l'emploi, à côté des termes officiels, de termes familiers, de xénismes et d'autres termes inacceptables dans un texte écrit. 
2. La rapidité de la transmission des messages en conférence, qui exige de l'interprète une grande vitesse de réaction et qui l'oblige à noter pour référence rapide non seulement les termes qu'il ne connaît pas, mais aussi les termes qu'il risque de ne pas trouver spontanément dans le feu de l'action, donc des termes en partie très simples appartenant à des domaines disparates, ainsi que des sigles, abréviations et noms propres qui ne lui serviront parfois que pour une seule conférence.

La préparation terminologique de l'interprète avant la conférence consiste essentiellement en la constitution de lexiques "maison " de quelques dizaines à quelques centaines d'entrées sur la base de l'exploitation des documents de conférence envoyés avant la réunion ainsi que d'autres documents portant sur les sujets devant être traités pendant la conférence. En effet, les dictionnaires et autres documents lexicologiques imprimés, relativement encombrants, lourds et dont les entrées sont sélectionnées selon certains critères d'économie, d'acceptabilité linguistique et d'homogénéité par domaines sont souvent moins bien adaptés au travail de l'interprète que des lexiques spécifiques légers et personnels constitués lors de la préparation, bien qu'ils aient également leur place à côté de ces lexiques.

\section{LA TERMINOTIQUE : LA FORMULE INDIVIDUELLE}

Compte tenu de la vitesse et de la facilité d'utilisation des systèmes de traitement de texte, ainsi que de la lisibilité des résultats mis en page et imprimés par l'ordinateur, les collègues interprètes équipés de micro-ordinateurs n'ont pas tardé à les exploiter pour leur préparation terminologique, en profitant notamment des avantages de la fonction "tri alphabétique»:

- Lors de la recherche d'《 équivalents » dans des dictionnaires, s'il a dressé préalablement une liste de mots en langue de départ triée par ordre alphabétique, l'interprète peut consulter les différents dictionnaires en tournant toujours les pages dans le même sens, sans retours en arrière. Ce détail apparemment banal fait gagner un temps considérable, et permet donc de préparer des lexiques plus complets que les lexiques manuels.

- La constitution manuelle de lexiques triés alphabétiquement par langues de départ est très longue et fastidieuse dès que le vocabulaire dépasse quelques dizaines de mots et deux langues, et ne se fait donc que rarement. Or, dans les lexiques non triés, la recherche des "équivalences" en cabine dans le feu de l'action est parfois très longue. L'informatique résout entièrement le problème en permettant à l'interprète d'entrer ses "équivalences"sans se préoccuper de leur mise en forme, puis de laisser l'ordinateur imprimer des listings triés pour chacune des langues concernées, ce qui réduit sensiblement le temps d'accès à chaque entrée lors de la recherche en cabine.

- Le tri met en évidence, en les alignant les uns après les autres, des " doublets " ou "triplets", termes apparaissant plus d'une fois dans un lexique avec éventuellement des "équivalents" différents, ce qui permet d'épurer le lexique en conséquence.

$\grave{A}$ un niveau d'organisation plus recherché, l'informatique permet de regrouper les termes dans les lexiques selon des groupes d'idées ou autres associations utiles.

Si l'informatique présente des avantages considérables dans le travail de préparation terminologique, ce qui se traduit dans la pratique par la constitution de lexiques plus complets et mieux organisés que les lexiques manuels, son apport potentiel en cours de conférence est plus considérable encore, mais à un niveau différent.

En effet, étant donné la rapidité de l'électronique, la recherche d'un terme technique par ordinateur ou terminal en cabine ne demande en principe que quelques secondes au maximum (nous mettons entre une et deux secondes pour composer un terme de dix mots sur un clavier, et un logiciel adapté devrait pouvoir trouver une entrée sur fichier très rapidement). La recherche manuelle est bien plus longue, et peut durer plu- 
sieurs dizaines de secondes s'il faut consulter plus d'un dictionnaire. Par ailleurs, la souplesse de l'ordinateur permet la fusion, le découpage, la réorganisation et la mise à jour immédiate de fichiers, et donne une grande puissance potentielle à l'outil informatique en cabine pour la préparation d'une intervention dont le texte vient juste d'être reçu ou pour la préparation ou la mise à jour de fichiers spécialisés en vue de conférences futures sur un même sujet.

À l'heure actuelle, ce potentiel évident n'est cependant pas réalisé. Si l'on s'appuie sur l'hypothèse que dans le cadre de la formule individuelle, les ordinateurs doivent pouvoir suivre l'interprète en cabine et donc être portatifs, l'on constate que sur le plan technique, les mémoires de masse disponibles sont encore trop fragiles (disquettes) ou trop lentes (cassettes de bande magnétique), et la mémoire vive encore restreinte, ce qui rend ces ordinateurs moins intéressants que les dictionnaires d'encombrement ou de poids équivalent : alors que des dictionnaires de format $\mathrm{A} 4$ et 3 à $4 \mathrm{~cm}$ d'épaisseur peuvent contenir plus de 150000 entrées, soit environ 6 Moctets de caractères, les ordinateurs portatifs les plus récents ont une RAM maximum de l'ordre de 500 Koctets et coûtent environ 50 à 100 fois plus cher. Quant aux quelques dizaines, voire 100 ou 200 termes qui correspondent aux lexiques préparés habituellement à l'occasion d'une conférence technique, ils tiennent en quelques feuilles de papier, dont la combinaison poidsencombrement-maniement les rend plus intéressantes que les ordinateurs. Il faudra donc attendre que les prix baissent et que la capacité des mémoires augmente pour que l'ordinateur trouve véritablement sa place en cabine, encore que quelques initiatives isolées aient déjà été prises dans ce sens (l'un de nos collègues utilise son Radio Shack modèle 100 en cabine en guise de lexique - voir Garcia Landa 1984, et nous-même utilisons occasionnellement le même appareil en cabine pour des mises à jour urgentes de fichiers terminologiques).

\section{LA FORMULE COLLECTIVE}

Toutefois, même avec des mémoires de masse et des mémoires vives adaptées et répondant aux besoins des interprètes en termes de capacité de stockage et de rapidité d'accès, l'interprète devra tout de même charger les données sur sa mémoire vive vierge. L'on peut envisager la commercialisation de "dictionnaires"sur support-mémoire à l'avenir, mais un travail de préparation individuel de la part de l'interprète restera néanmoins nécessaire étant donné la spécificité de chaque conférence; nous pensons toutefois que la formule collective des réseaux, qui connaît actuellement un grand essor dans le monde de l'informatique, est susceptible de multiplier par un facteur non négligeable la puissance de l'outil informatique dans le travail terminologique de l'interprète. En effet, les réseaux permettent d'une part des échanges d'informations avec des collègues ayant déjà travaillé dans un même type de réunion, et d'autre part la consultation de bases de données constituées, telles qu'il en existe déjà plusieurs, notamment en Europe et au Canada. Au cours de son travail de préparation, l'interprète relié à un réseau pourrait donc charger des sous-ensembles de lexiques et bases de données constitués par ses collègues, puis les modifier et les compléter en fonction de ses besoins spécifiques. Pour des raisons évoquées plus haut, le contenu des bases de données terminologiques existantes est sélectionné et mis à jour selon des critères qui ne répondent pas à l'ensemble des besoins des interprètes, bien qu'il en couvre une partie non négligeable. C'est pourquoi il nous semble que la meilleure formule pour l'option collective consisterait en la possibilité d'utiliser en même temps ces bases de données et des bases de données spécifiquement destinées à l'interprétation, ce qui ne manquera pas de poser des problèmes : problèmes techniques si l'on veut préserver la rapidité d'accès requise, et problèmes de gestion de l'information, celle-ci étant susceptible d'" exploser» en peu de temps avec 
l'apparition de bases de données, de bases de données modifiées, de sous-bases de données multiples, modifiées puis fusionnées, soit une masse de bases de données et de fichiers dont le contenu se recouperait en grande partie.

\section{LA SITUATION ACTUELLE}

La formule collective de l'utilisation de l'informatique n'est pour l'instant pas matérialisée, si l'on exclut l'utilisation par les interprètes de lexiques informatisés préparés pour les traducteurs ou terminologues. D'ailleurs, la matérialisation de la formule personnelle est elle aussi fort récente. En fait, il y a quatre ans encore, le nombre d'interprètes de conférence équipés d'ordinateurs était très faible. Par la suite, la vague déferlante des micro-ordinateurs personnels a atteint les interprètes, qui se sont vite rendus compte du potentiel que l'informatique représentait pour leur travail de préparation terminologique.

Petit à petit, l'on s'est donc équipé, avec dans l'ensemble des matériels assez lourds (IBM-PC, Apple, MacKintosh, etc.) et des logiciels de traitement de textes et de bases de données perfectionnés. Nous pensons personnellement qu'étant donné les applications actuelles (traitement de textes et lexiques de quelques centaines de mots au maximum), il est possible d'arriver à des résultats identiques ou très proches avec un matériel très peu coûteux (voir Gile 1983) et des programmes "maison " : il suffit en effet d'une fonction tri et d'une fonction " permutation " pour réaliser des lexiques de plusieurs centaines de termes sur un matériel très modeste (voir Annexe).

Au-delà des tentatives d'expérimentation menées par des interprètes isolés, Francis Leclercq, membre de l'Association internationale des interprètes de conférences, s'est dépensé en efforts de sensibilisation, d'information et de coordination qui ont abouti à la création d'un Groupe de réflexion sur l'informatique personnelle (GRIP) au sein de l'Association. Ce groupe a préparé récemment à l'intention des collègues intéressés une feuille de recommandations pour les interprètes désirant s'équiper. Les regards commencent d'ailleurs à se porter plus loin, vers des thèmes tels que la traduction et l'interprétation assistées par ordinateur, la gestion des calendriers des interprètes, la gestion de l'Annuaire de l'AIIC, etc.

\section{PERSPECTIVES D'AVENIR}

Vue dans la perspective de l'interprétation de conférence, l'évolution technologique devrait permettre de construire des ordinateurs portatifs que leur capacité rendra compétitifs par rapport aux dictionnaires et lexiques sur papier, ce qui rendra plus intéressante la « formule individuelle». Il nous semble toutefois que seule la formule collective, et notamment la mise en place de réseaux importants, permettra d'exploiter plus pleinement le potentiel de l'informatique. Les problèmes techniques et d'organisation liés à ces réseaux ne seront pas faciles à résoudre, d'autant que le marché des interprètes de conférence est très limité et ne justifie pas de gros investissements de la part des sociétés commerciales. L'on peut probablement s'attendre à l'utilisation de réseaux commerciaux avec des logiciels commerciaux, et parallèlement à la mise en place de bases de données terminologiques artisanales qui seront très difficiles à gérer. Il faudra notamment déterminer assez rapidement, dès que les premiers éléments de réseaux se mettront en place, des critères de sélection, de maintien et d'élimination, sous peine de se retrouver rapidement confronté à une inondation terminologique incontrôlable.

L'enjeu n'est pas négligeable, car la compréhension et la connaissance des termes spécialisés peuvent apporter à la qualité du travail en interprétation de conférence un incrément très important. C'est pourquoi l'on suivra avec intérêt l'évolution de la situation. 


\section{RÉFÉRENCES}

GARCIA-LANDA, M. (1983) : L'ordinateur de cabine au banc d'essai ", Circuit, organe de la Société des traducteurs du Québec, septembre.

GILE, D. (1983) : "Les petits lexiques informatisés : quelques réflexions", Bulletin de l'AIIC, XI-3, septembre.

GILE, D. (1985a) : "L'analyse dans la traduction humaine", Actes de COGNITIVA 85, Paris, juin.

GILE, D. (1985b) : "Les termes techniques en interprétation simultanée ", META, 30-3, septembre.

GILE, D. (1985c) : "Le travail terminologique en interprétation de conférence", Multilingua, 4-3.

\section{ANNEXE \\ Éléments de base pour la "formule individuelle"}

À l'intention des collègues qui souhaitent « informatiser » leur préparation terminologique à faible coût, les lignes suivantes décrivent une formule minimum que nous employons avec un Radio Shack modèle 100 (32 K de RAM) pour la préparation de lexiques de plusieurs dizaines à 200 ou 300 entrées environ. Les lexiques entrent entièrement en RAM - ils sont sauvegardés sur cassette.

La formule choisie, destinée à la préparation de lexiques bilingues, se fonde sur trois éléments :

1. des fichiers de textes,

2. un programme de tri alphabétique,

3. un programme de permutation maison.

Les fichiers de textes font partie de tous les logiciels de traitement de textes. On trouve aussi des fonctions de tri alphabétique sur de tels logiciels, mais il est également possible de recopier l'un des nombreux programmes de tri figurant dans de nombreux livres d'informatique. Le seul élément "original " parmi les trois est le petit programme de permutation qui transforme les entrées " terme en langue $A=$ terme en langue $B$ " en "terme en langue $B=$ terme en langue $A$ », et qui peut être aisément adapté à une application trilingue ou quadrilingue.

La préparation des lexiques bilingues se fait de la manière suivante :

- Les entrées sont écrites sur fichier " texte "à mesure qu'elles apparaissent, toujours dans le sens "Langue $A=$ Langue $B$ ".

- Une fois le fichier fermé, la fonction tri est appelée, et son déclenchement donne un lexique langue $\mathrm{A}-$ langue $\mathrm{B}$.

- Après impression et sauvegarde de ce fichier sur cassette, la fonction permutation est appelée, et transforme le fichier "langue $A$ - langue $B$ » en fichier " langue $B-$ langue $A$ " non trié.

- La fonction " tri » est appelée une deuxième fois, et donne un lexique « langue B - langue A » trié.

En réalité, des fonctions supplémentaires facilitant la lecture et la mise à jour des fichiers ainsi que l'extraction de sous-fichiers ont été également mises au point, mais l'essentiel opérationnel tient dans les trois éléments évoqués plus haut.

La routine de permutation que nous avons écrite en BASIC (voir ci-dessous) est très simple : chaque entrée $\mathrm{D} \$(\mathrm{I})$ étant composée d'un terme en langue $A$, puis d'un blanc, puis du signe " =", puis d'un blanc, et enfin d'un terme en langue $\mathbf{B}$, la routine cherche le signe " = (ligne 3020). S'il n'y en a pas, elle ne change rien à la ligne, ce qui permet de garder telle quelle une entrée de fichier correspondant à un terme sans équivalent, à un titre ou à une autre indication qui ne doit pas être permutée. Si l'entrée comporte un « = ", la routine définit comme $\mathrm{A} \$$ le terme en langue $\mathrm{A}$ et comme $\mathrm{B} \$$ le terme en langue $B$ (lignes 3100 et 3250 ), puis elle remplace l'entrée $D \$(I)$, qui a initialement la forme " $\mathrm{A} \$=\mathrm{B} \$$ ", en " $\mathrm{B} \$=\mathrm{A} \$$ " (ligne 3500). 


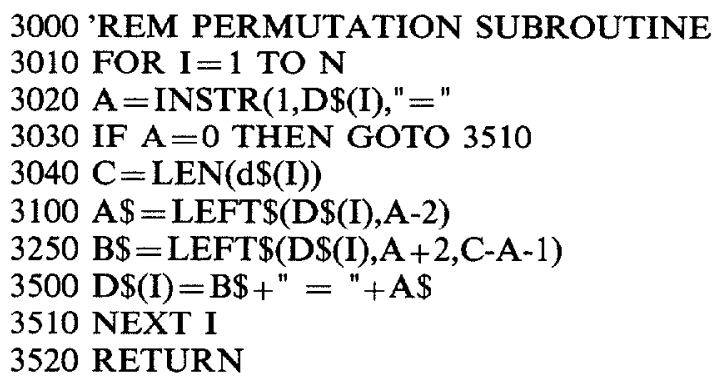

Cette routine est facile à adapter à d'autres dialectes de BASIC et à intégrer à un programme qui chercherait un fichier de texte, l'" ouvrirait " puis le fermerait après manipulation. 